Note to readers. For the definitive version of this article, view :

Chase, J.G., Lambermont, B., Starfinger, C., Hann, C.E., Shaw, G.M., Ghuysen, A., Kolh, P., Dauby, P.C. and Desaive, T. (2011) Subject-specific cardiovascular system model-based identification and diagnosis of septic shock with a minimally invasive data set: Animal experiments and proof of concept. Physiological Measurement, 32(1), 65-82.

\title{
Subject-specific cardiovascular system model-based identification and diagnosis of septic shock with a minimally invasive data set: \\ Animal experiments and proof of concept
}

J.Geoffrey Chase ${ }^{(1)}$, Bernard Lambermont ${ }^{(2)}$, Christina Starfinger ${ }^{(1)}$, Christopher E. Hann ${ }^{(1)}$, Geoffrey M. Shaw ${ }^{(3)}$, Alexandre Ghuysen ${ }^{(2)}$, Philippe Kolh ${ }^{(2)}$, Pierre C. Dauby ${ }^{(2,4)}$ and Thomas Desaive ${ }^{(2,4)}$

(1) Centre for Bioengineering, University of Canterbury, Christchurch, New Zealand

(2) Cardiovascular Research Center, University of Liege, Belgium

(3) Department of Intensive Care Medicine, Christchurch Hospital, Christchurch, New Zealand

(4) Institute of Physics, University of Liege, Belgium

Running head: Subject-specific modelling of the cardiovascular system

\author{
Contact information: \\ Corresponding author: Thomas Desaive \\ Corresponding address: Dr Thomas Desaive \\ University of Liege \\ Institute of Physics, B5 \\ Allee du 6 aout, 17 \\ 4000 Liege \\ Belgium \\ e-mail address: tdesaive@ulg.ac.be
}




\begin{abstract}
A cardiovascular system (CVS) model and parameter identification method have previously been validated for identifying different cardiac and circulatory dysfunctions in simulation and using porcine models of pulmonary embolism, hypovolemia with PEEP titrations, and induced endotoxic shock. However, these studies required both left and right heart catheters to collect the data required for subjectspecific monitoring and diagnosis - a maximally invasive data set in a critical care setting although it does occur in practice. Hence, use of this model-based diagnostic would require significant additional invasive sensors for some subjects, which is unacceptable in some, if not all, cases. The main goal of this study is to prove the concept of using only measurements from one side of the heart (right) in a "minimal" data set to identify an effective patient-specific model that can capture key clinical trends in endotoxic shock.
\end{abstract}

This research extends existing methods to a reduced and minimal data set requiring only a single catheter and reducing the risk of infection and other complications - a very common, typical situation in critical care patients, particularly after cardiac surgery. The extended methods and assumptions that found it are developed and presented in a case study for the patient-specific parameter identification of pig-specific parameters in an animal model of induced endotoxic shock. This case study is used to define the impact of this minimal data set on the quality and accuracy of the model-application for monitoring, detecting and diagnosing septic shock.

Six anesthetized healthy pigs weighing $20-30 \mathrm{~kg}$ received a $0.5-\mathrm{mg} / \mathrm{kg}$ endotoxin infusion over a period of 30 mins from T0 to T30. For this research, only right heart measurements were obtained. Errors for the identified model are within $8 \%$ when the model is identified from data, re-simulated and then compared to the experimentally measured data, including measurements not used in the identification process for validation. Importantly, all identified parameter trends match physiologically and clinically and experimentally expected changes, indicating that no diagnostic power is lost.

This work represents a further with human subjects validation for this model-based approach to cardiovascular diagnosis and therapy guidance in monitoring endotoxic disease states. The results and methods obtained can be readily extended from this case study to the other animal model results presented previously. Overall, these results provide further support for prospective, proof of concept clinical testing with humans.

Keywords: cardiovascular system, cardiac model, parameter identification, integral method, endotoxin, septic shock 


\section{Introduction}

Sepsis is a very complex and serious systemic response to infection. Sepsis results in as many deaths in the USA as out-of-hospital cardiac arrests, and four times the number for breast cancer [1]. More specifically, mortality rates have ranged from $25 \%$ to $80 \%$ over the last few decades [2]. Septic shock or severe sepsis and multiple organ failure are thus one of the leading causes for morbidity and mortality in the critical care setting.

Septic shock is characterized by a decreased venous return to the right heart caused by the combinatory effects of capillary leaks, reduced stressed systemic volume and loss of circulatory tone, leading also to a decreased systemic arterial resistance. Moreover, venous capacitance and pulmonary resistance are usually increased, which, together with a reduced ventricle contractility, lead to impaired stroke volume and cardiac output [3]. A simultaneously decreased systemic arterial resistance therefore leads to a low blood pressure and consequently hypotension. Fluid resuscitation can compensate for the capillary leak and result in increased venous return. Most patients in septic shock after fluid resuscitation thus have a high cardiac output, but a low systemic vascular resistance state [4-6] and by definition all need vasopressors to keep mean systemic arterial pressure in normal range.

These points thus clearly indicate the key clinically important diagnostic characteristics and treatments, as well as the need to obtain an early diagnostic as soon as the dysfunction begins to occur to provide the greatest chance for successful intervention. In addition, it is clearly evident that the primary therapies have a feedback effect on the cardiovascular system that can be difficult to predict. Hence, model-based methods for patient specific diagnosis of this dysfunction and others have been developed.

In particular, the CVS model and identification process used herein have previously been validated in the identification and model-based analysis of induced endotoxic shock with continuous veno-venous hemofiltration $(\mathrm{CVVH})$ therapy in a porcine animal model [7]. It has also been applied to similar porcine animal model analyses of pulmonary embolism [8] and mechanical ventilation in ARDS [9, 10]. In this research, a porcine model of induced endotoxic shock without hemofiltration is analyzed and the CVS 
model parameters are identified.

The novelty of this identification is that for the first time the identification process is applied to strictly right ventricle signals and no left ventricle signals were measured. This significantly reduced data set is of particular clinical importance, as often only limited data, such as data from only one of the ventricles, is available. Reducing this data set reduces the number of highly invasive catheters required from an unusual in critical care value of two, to a very typical value of one catheter. Hence, it also reduces significant added risk of complications and infection that can be associated with these catheters [11]. This analysis on the impact of the reduction of invasive catheters is thus critical to establishing the potential and initial feasibility of this model-based approach to monitoring and diagnosis of cardiovascular dysfunction using experimental animal models of endotoxic shock.

More specifically, the CVS model and identification process are therefore applied here to only the measured right ventricle signals under a series of assumptions. However, similar contractility and afterload trends are obtained when compared to previously reported experimental results using full data sets for both the left and right heart [12]. The overall goal is to show the robustness of the extended methods developed, to define the clinical potential to use this type of minimally invasive, model-based physiological monitoring to diagnose clinically important developing disease states.

\section{Methodology}

\section{CVS model}

The CVS model is a lumped parameter model, previously developed by $[13,14]$ and based on earlier simplified models with less chambers and variables $[15,16]$. The new and extended model is presented in detail in $[9,10,17]$. Briefly, the new and extended CVS model includes one extra compartment for the lung capillaries and a second, extra compartment has been added for the systemic capillaries. This change separates the venous and arterial systems and resistances, which is more physiologically accurate. Figure 1 shows these modifications with the two new compartments for the systemic and pulmonary capillaries. 
This physiologically relevant addition is of great interest as two resistances on either side, the arterial and venous side, are necessary to correctly model/capture the complex behaviors observed clinically during, for example, mechanical or spontaneous breathing [10], thus physiologically justifying this model extension based on results from an independent clincial scenario and independent set of data.

This new model also more correctly represents the anatomy, where the pulmonary arteries and veins as well as the vena cava are within the thoracic cavity. Moreover, neither the aorta, as modelled as part of the systemic circulation, nor the lung capillaries, which are surrounded by alveolar pressure, are surrounded by intrathoracic pressure $\left(\mathrm{P}_{\mathrm{th}}\right)$. More details about this new model definition and its clinical and physiological relevance are available in $[7,9,10,17]$ and the main equations are recalled in the appendix.

\section{Parameter identification method}

In this research, the main goal is to identify the CVS model parameters based only on measured input data from the right side of the heart, using only estimated left ventricle signals. The measured signals for this research that are used for identification are the pressures in the aorta and pulmonary artery $\left(\mathrm{P}_{\mathrm{ao}}, \mathrm{P}_{\mathrm{pa}}\right)$, and the volume in the right ventricle $\left(\mathrm{V}_{\mathrm{rv}}\right)$.

The complete identification process using a full set of measured data and catheters in both the left and right heart, has previously been described [7-10]. Therefore, in this research, only a brief overview is given focusing on the specific aspects of performing the identification with the reduced data set. In particular, as the left ventricle signals are not measured in this reduced data study, they must be estimated for the identification process, which is thus the focus and novelty of this approach presented.

For this study, the left ventricular volume $\left(V_{\mathrm{IV}}\right)$ is assumed to be the same as the measured right ventricular volume $\left(\mathrm{V}_{\mathrm{rv}}\right)$ for simplicity. Thus, the left ventricle stroke volume (LVSV) is also assumed to be the same as the measured right ventricle stroke volume (RVSV). The left ventricular pressure $\left(P_{\mathrm{lv}}\right)$ is not required during the identification process and is thus not specifically estimated. These approximations are 
not necessarily completely accurate in a dynamic, septic patient or animal model thereof, but provide a useful, effective and physiologically justified initial starting point.

The waveforms are artificially generated by scaling a set of previously calculated model outputs to best fit the measured maximum and minimum data values for the pressures and volumes. The assumption is that these model waveforms are reasonably conformable with the actual case seen clinically. In addition, it is thus assumed that thse signals are representative of the model's ability to capture any similar waveform. This approach is similar to others taken in the literature with similarly minimal data sets [18-21].

These scaled signals are then used in place of the (now unmeasured in this study) left heart signals in the full parameter identification process reported previously. Subsequent to this identificaiton a new CVS forward simulation is performed with theses newly identified parameters. The result is a much closer match to the experimental data than what was provided by the first initial parameter set. This animalspecific model simulated output is then compared to the experimental data to assess performance.

In further subsequent iterations, these new model-generated output signals can be re-scaled again, and a subsequent (second or higher) set of model parameters identified. The results of this subsequent set of parameter identification interations are (as above) used to run a further simulation. This overall iterative process is stopped when the relative error between model output and measured experimental data reaches a set tolerance or fails to improve. Figure 2 gives an overview of the overall identification process.

Model parameter values are presented as mean normalized values over all analyzed pigs to allow comparison between pigs who have very different values for an equivalent physiological state. Previously reported values for the same experimental data of induced septic shock [12] are also normalized for comparison. Data values are normalized with regard to the baseline (time $=0$ mins) value for each pig to guarantee the correct representation of the parameter trends over all pigs, rather than showing the absolute values where extreme low or high values for one pig can easily influence the mean value over all pigs. All the data is thus self-normalized before comparison across pigs or treatment method. 


\section{Volume calculations}

The central venous pressure (CVP) or systemic pressure $\mathrm{P}_{\text {sys }}$ plays a central role in the CVS model and identification process. The CVP provides insight into the venous system function and, more specifically, about the effective systemic volume $V_{\text {sys,eff. }}$ However, CVP is not measured in this experiment and there is also no directly measured information available about the volume status of the pigs. Therefore, certain assumptions about the volumes and pressures in the CVS model have to be made.

These assumptions help guarantee that the best and most optimal parameters, relative to these assumed volumes, are identified. In all cases, the identified model parameters are then used to re-simulate the model and produce output signals that not only match the measured signals, but also all other estimated volumes and pressures. The overall approach thus provides a consistent means of identification and method validation, given the limited available experimental data in this study, which is representative of a typical clinical scenario. Hence, while specific values for unmeasured (left ventricle) pressures and volumes may not be perfect based on these assumptions, the main goal is to ensure key clinical trends of the identified model parameters match those expected in experimentally observed endotoxic shock.

More specifically, it is known that during septic shock the effective systemic volume decreases as a result of capillary leak (absolute hypovolemia) or venodilation (relative hypovolemia) [5, 6]. Therefore, the evolution of septic shock was simulated in this research by a stepwise reduction of $5 \%$ of the effective systemic volume $\mathrm{V}_{\text {sys,eff }}$ every 30 minutes. This stepwise reduction is a rather conservative estimate of the reduction in volume [22]. If necessary, the CVS model can easily be run with more severe reductions simulating more severe forms of septic shock. Hence, septic shock may be assessed parametrically in clinical or experimental situations by testing the model and identificaiton for such reductions, although that is not tested here. Thus, in this study, all initial CVS model volumes are estimated based on the known distribution of blood, as previously described in $[9,10]$.

\section{Experimental protocol}


All experimental procedures and protocols used in this investigation were reviewed and approved by the Ethics Committee of the Medical Faculty of the University of Liege. The investigation conforms with the Guide for the Care and Use of Laboratory Animals published by the US National Institutes of Health (NIH Publication No. 85-23, revised 1996).

Experiments were performed on 6 healthy pure pietran pigs of either sex weighing from 20 to $30 \mathrm{~kg}$. The animals were premedicated with intramuscular administration of ketamine (20 mg/kg) and diazepam (1 $\mathrm{mg} / \mathrm{kg})$. Anesthesia was then induced and maintained by a continuous infusion of sufentanil $(0.5 \mu \mathrm{g} / \mathrm{kg} / \mathrm{h})$ and pentobarbital $(5 \mathrm{mg} / \mathrm{kg} / \mathrm{h})$. Spontaneous movements were prevented by pancuronium bromide $(0.2$ $\mathrm{mg} / \mathrm{kg}$ ). After endotracheal intubation through a cervical tracheostomy, the pigs were connected to a volume cycled ventilator (Evita 2, Drager, Lubeck, Germany) set to deliver a tidal volume of $10 \mathrm{ml} / \mathrm{kg}$ with a $\mathrm{FiO}_{2}$ of 0.4 and at a respiratory rate of 20 breaths /min. End-tidal CO2 (PETCO2 ) measurements (Capnomac, Datex, Helsinki, Finland) were used to monitor the adequacy of ventilation. Respiratory settings were adjusted to maintain PETCO2 between 30 and $35 \mathrm{mmHg}$.

The animals received a $0.5 \mathrm{mg} / \mathrm{kg}$ endotoxin infusion (lipopolysaccharide from Escherichia coli serotype 0127:B8; Sigma Chemical, St. Louis, MO, U.S.A.) over 30 mins (from T0 to T30) and received no further intervention. Measurements were obtained for systemic arterial pressure $\left(\mathrm{P}_{\mathrm{ao}}\right)$, pulmonary arterial pressure $\left(P_{p a}\right)$ and right ventricle pressure and volume $\left(P_{r v}, V_{r v}\right)$ as described previously in [12].

Measurements were obtained every 30 minutes into the experiment, from T0 to T300 minutes. Note, that for pig 1, only 4 measurements were obtained (T0 - T90); for pig 2, 9 measurements (T0 - T240); for pig 5, 8 measurements (T0 - T210) and for pig 6, 7 measurements (T0 - T180). Pigs 3 and 4 had the full amount of 11 measurements (T0 - T300), thus totalling 50 measurements over all pigs. 


\section{Analysis of Results:}

The main form of analysis is comparison of errors at each time point in the experiment between the resimulated animal specific model and the experimental data, where, as noted, the right ventricular pressures were not used in the identification process and provide a unique further form of validation. Further, each time point in this experiment is a specific cardiovascular state that is uniquely different from the prior one as endotoxic shock progresses. Therefore, comparisons of errors or fitting between time points in the progression of endotoxic shock, except as presented in aggregate in Table 1, are less relevant as they represent very different physiological states that the model may or may not capture. In addition, unavoidable variability in the progression of experimental endotoxic shock will make further comparisons even within time periods difficult. What is more important in this proof of concept analysis is to determine if the errors are consistent across all pigs in each specific state to identify if there are specific physiological states the model cannot identify good parameters or trends.

Each identified value for all major signals is therefore plotted for each pig at all time points captured for that pig, clearly showing the errors for that time point and pig in progression. These plots are concatenated to show: a) all 6 pigs and errors on a single plot for clarity; and b) to show the variability in physiological signals seen across pigs in each state. Both of these factors would be lost by lumping all errors and states together.

Finally, there are very few overall points $(\mathrm{N}=59)$ which, considering 5 major time points and physiological states in the experiment with very different values for these identified signals. Hence, obtaining more than a central tendency (median and IQR) is not effective to show the shape or distribution that might arise for greater numbers. These values are already plotted as noted above. Finally, non-parametric statistics are used (median, IQR) due to the relatively uniform (non-Gaussian) distribution of errors around median values. 


\section{Results}

Figure 3 (a)-(c) illustrates the match between experimental data and identified and re-simulated model for one specific pig in detail at 0,90 and 120 minutes. The first subfigure shows the right ventricle signals at the beginning of the experiment (TO). The upper panel shows the experimental vs. simulated right ventricular pressure and the pressure in pulmonary artery. In the lower panel, the experimental vs. simulated right ventricular volume is shown. Importantly, during the identification process only the systolic (maximum) and diastolic (minimum) values of the measured ventricle volume (EDV, ESV) and arterial pressure (SPAP, DPAP) are used. The right ventricular pressure (Prv) is not used, as this measurement is rarely obtained in a typical clinical setting, although it is more common in some research settings and was thus measured in this study. Thus, it is important to note that the RV pressures (top plots) in Figures 3 have very little error, as confirmed in Table 1 for all pigs with median absolute error of $2.87-4.33 \%$ for systolic and diastolic pressures, even though that measurement was not used in the identification process. This "true" validation should further serve to show the capability of the identification process and model.

If desired, the ventricle pressure waveforms between the minimum and maximum values could easily be matched more accurately by adjusting the generic activation driver function shapes used in the CVS model $[13,14]$. However, this level of accuracy and added modification was not intended in this study. Additionally, clincal diagnostics are driven primarily by these minimum and maximum values, and their range, and not by te specific waveform shape. The following subfigures illustrate the same results and level of correlation between model and data at 90 and 120 minutes into the experiment for the same pig as in Figure 3.

As noted, it was never intended to perfectly match the pressure and volume waveform shapes, but only the minimum and maximum experimentally measurable values, and to thus capture key cliincal trends. This goal was adopted because the main focus is to identify the overall macro-hemodynamic condition, and less interest is placed on exactly matching the specific waveforms. 
Table 1 shows the median absolute percentage errors for the identified minimum and maximum pressure and volume signals (SAP, DAP, SPAP, DPAP, RVESV, RVEDV, RVSV) for the identified re-simulated model over all pigs. It also shows the span of the interquartile range $\left(75^{\text {th }}\right.$ percentile $-25^{\text {th }}$ percentile $)$ as it was centered uniformly (non-Gaussian) around the median value in all cases for this analysis. The central tendency (median, IQR) of the errors is well below 10\%, which is within typical measurement noise levels.

Figure 4 shows the right ventricular stroke volumes (RVSV) for all identified time segments over all pigs. The solid line represent the experimental data while the crosses represent the CVS model simulation output when re-run using the animal-specific identified model parameters. As can be seen, the model output values match the true experimental values very well, with median absolute percentage errors less than $5 \%$, which is well within measurement or estimate errors $[23,24]$. It can also be seen that there is significant inter-pig variation, as might be expected, particularly for pig 2 that has a much higher set of values. Hence, this results also shows the model's ability to capture, in a pig-specific fashion, the potentially significant and large variation between subjects that could affect diagnostic accuracy if missed.

Figure 5 shows the systemic arterial systolic and diastolic pressure values (SAP, DAP) over all times and pigs. The solid lines represent the experimental measurement and the,$+ \square$ markers represent the identified animal-specific CVS model output for SAP and DAP respectively. Again, per the values in Table 1, matches are obtained with median absolute percentage errors less than $3 \%$ and minimal IQR (centered on the median) range of less than $5 \%$, which are below measurement errors of $10 \%$ for the same signals.

Figure 6 shows the pulmonary arterial systolic and diastolic pressure values (SPAP, DPAP) over all times and pigs. The solid lines represent the experimental measurement and the,$+ \square$ markers represent the identified, animal-specific CVS model output for SPAP and DPAP respectively. Matches with similar error in Table 1 are obtained, with median absolute percentage errors less than $7 \%$.

Figure 7 (a)-(d) shows the mean normalized systemic stressed volume $\left(V_{\text {sys,eff }}\right)$, and the identified mean normalized systemic vascular $\left(R_{\text {sys }}\right)$ and pulmonary resistance $\left(R_{\text {pulin }}\right)$, as well as the resistance to venous 
return $\left(R_{\mathrm{vr}}\right)$ over all pigs during the endotoxic shock experiment.

The upper panel in Figure 8 shows the experimentally measured right ventricular end-systolic elastance $\left(E_{\text {esrvf }}\right)$ during the endotoxic shock experiment, as previously reported [12]. The lower panel of Figure 8 shows the similar results obtained from the CVS model and identification process using the limited data set available for this study. This elastance, as with other model parameters, is found in the parameter idnetification, and its diagnostic valuae is independently assessed using clinical calculations [12].

Note that the absolute values obtained for $E_{\text {esrvf }}$ depend on the unstressed ventricle volume $\left(V_{d}\right)$. For reasons of simplicity, this value is assumed to be zero $\left(\mathrm{V}_{\mathrm{d}}=0 \mathrm{ml}\right)$ during the identification process. However, $E_{\text {esrvf }}$ can easily be adjusted for a different, and probably more realistic, $V_{d}$, as these adjustments have no effect on the other identified model parameters. Overall, the identified $\mathrm{E}_{\mathrm{esrvf}}$ trends match well with recently reported values obtained for the same experimental data [12], as shown in comparing the upper and lower panels of Figure 8.

\section{Discussion}

One of the major goals of this research is to show the applicability of the CVS model and identification process to capture key experimentally observed trends over time for a clinically difficulty and important scenario using a severely reduced, minimally invasive data set that is clinically typical/relevant. In particular, in this research, only the right heart signals $\left(V_{r v}, P_{p a}\right)$ and the arterial pressure $\left(P_{a o}\right)$ are available for identifying the induced endotoxic shock. Despite this restricted data set and the additional assumptions made regarding the other model volumes and pressures, the CVS model parameters are consistently identified. This accurate level of identification is validated by re-simulation and comparison to experimental data including measured data not used in the identification process. Importantly, it holds true not only for the right part of the model, but for the entire parameter set.

When re-simulating the model with the identified parameters, the output signals produced match the observed experimental data very well, as shown in Figures 4, 5 and 6. Median absolute percentage errors 
are less than $5 \%$ for the volumes and less than $7 \%$ for the pressures, as shown in Table 1 . These errors are well within typical experimental measurement errors $[23,24]$.

A second important finding of this research is that, despite the reduced data set and necessary assumptions made, the CVS model is able to accurately identify and re-simulate endotoxic shock. Clinically, and importantly, the identified parameter trends match physiological expectations. For example, Figure 7 shows the model simulated normalized systemic volume and how it decreases during the time course of the experiment, accounting for the loss of circulatory control and hypovolemia seen in septic shock $[5,6]$. The data and results obtained from the pig-specific identified models thus match clinical and experimental measurements and expectations.

Figure 7 also shows the mean normalized identified trends for the CVS model parameters $R_{\text {sys }}, R_{\text {pulin }}$ and $R_{\mathrm{vr}}$ over all analyzed pigs during the experiment. The value of $R_{\text {sys }}$ is perhaps (unexpectedly) identified to initially increase at the beginning of the experiment. However, after $30 \mathrm{~min}$ and with the ongoing endotoxin infusion, $\mathrm{R}_{\text {sys }}$ is decreasing, with a further two relatively small increases at the end of the experiment. Overall, this trend indicates an initial physiological compensation followed by a failure of circulatory control that is typical of septic shock [6]. From a model-based diagnostics perspective, this result accurately tracks disease onset, initial (successful) compensation and (eventual) physiologic failure, all of which are clinically significant in determining appropriate therapies and dosing.

The mean normalized $R_{\text {pulin }}$ values over all pigs increase during the experiment, as expected $[5,6]$. There is a sharp drop at 60 mins into the experiment. However, this decrease is consistent with previously published results [12], where a similar drop can be observed at 60 min. Clinically, the model is thus capturing the known effects of sepsis in this portion of the circulation, as well.

Given the potential clincal use of this model-based monitoring and diagnostic method, it is important to note that differences in systemic $\left(R_{\text {sys }}\right)$ and pulmonary $\left(R_{\text {pulin }}\right)$ behaviours and resistances can help dictate the clinical therapeutic balance between vasopressor and fluid resuscitation therapy if they are known. 
Hence, this model and identification method can provide this level of clinical advice and guidance. Equally importantly, it has accomplished this task in this study despite significant inter-pig variation in measured data, as well as significant intra-pig variation over time as the induced septic shock evolves and impacts cardiovascular system function.

Diagnostically, the mean normalized resistance to venous return, $\mathrm{R}_{\mathrm{vr}}$, decreases consistently during the experiment. This result is expected, as the venous return is already impeded by a low blood volume caused by fluid losses into the tissue, as well as by the effects of veno- and vasodilation. In septic shock, proinflammatory cytokines cause an increase in endothelial-derived nitric oxide, which in turn is a major mediator for vasodilation and hypotension [5]. Therefore, the body tries to restore venous return and the model identifies this behaviour as a lowered resistance to venous return that is needed to compensate for the lower venous volume, pressure and flow back to the heart. Again, this capability to very specifically capture the circulatory dysfunction due to sepsis has significant importance in the realms of clinical diagnosis and therapeutic decision support required to first identify the dysfunction in a timely fashion and to then balance therapy selection and dosing.

The model makes several key assumptions due to the clinically realistic, but otherwise minimal, data set utilised. Notably, that the volumes of the left and right ventricles, as well as their stroke volumes, are equal. The quality of the results rests on the validity of these assumptions over the cases observed and typical behaviours in critical illness.

First, the more critical assumption that the left and right ventricle stroke volumes are equal is axiomatically valid in virtually every typical circumstance [25]. The exception to this case is when there is significant, measurable intra-cardiac shunt. However, this case occurs very rarely and is due to either an acquired condition, such as a septal rupture following an acute myocardial infarction, or because of a congenital heart condition. However in those rare circumstances other clinical signs or results of investigations would usually identify these conditions a priori. Hence, this assumption is valid and supports the use of only a single catheter in the practical, clinical application of this approach. 
Given this limitation, a comparison can be drawn to prior work $[7,26]$ that had a more complete data set across both ventricles. Although the data sets did not allow direct analysis or comparison (by withdrawing measurements and repeating). The median errors for common identified variables can be contrasted. Specifically, the errors for [SAP SPAP RVEDV RVESV] $=\left[\begin{array}{ll}2.87 & 3.02 \\ 1.37 & 1.55\end{array}\right]$ in Table 1, and are [3.09 $7.011 .181 .90] \%$ in $[7,26]$. Although the experiments were quite different in specifics, the very similar values, and median errors below the $8-10 \%$ expected of measurement noise across similar numbers of pigs indicates that very little has been lost in this reduced data set. What has been lost more specifically, is the ability to obtain animal-specific left heart parameters as in $[7,26]$. Thus, the main difference is one of identifying less parameters, rather than an increase in error.

Second, is the less critical assumption of equality between left and right ventricle volumes. This assumption is not always true at a given point in time due either to normal physiological differences in cardiac anatomy where the left ventricle is slightly larger, or to specific patient condition such as pulmonary embolism. However, its assumption over a given analysis, as presented here, does not detract from the results. Importantly, the relatively small $(<15 \%)$ differences in volumes that occur merely shift the results obtained in identifying the model. However, it is very important to note that the monitoring and diagnostic importance of the model lies in the trends obtained for model parameters over the course of a disease state and not in the specific values identified. These trends are valid even if the ratio between ventricular volumes is different from that assumed.

To test both these issues and assumptions, particularly in humans, would require, in most cases, more invasive monitoring than would be justified clinically. In particular, it would require that both sides of the heart have an appropriate catheter to assess stroke volume, and further invasive measurements or echocardiography to assess ventricular volume. Such trials would be ethically challenging in humans, and thus not likely warranted unless these assumptions were seriously questioned by adverse results in upcoming human clinical trials. 


\section{Conclusions}

The extended integral-based parameter identification approach presented successively identified pigspecific parameters for the extended CVS model using a significantly reduced and clincally relevant set of measurements. This further validation study shows the ability of the model to adequately and realistically capture the impact of pressure-volume changes during endotoxic shock. Robustness was demonstrated in the ability to capture significant intra- and inter- pig variation, while clincial validation was evident in the ability of the identified model to capture measurements that were made but not used in the identification process. Finally, all results matched the measured (used and unused) experimental data within typical measurement errors, as well as capturing all expected physiological and clinically expected trends, indicating its clinical suitablility as a real-time diagnostic tool. In particular, the model is able to aggregate diverse measured data into a clear, clinically and physiologically relevant diagnostic picture as the condition develops, and can do so with a clinically relevant and minimal data set with respect to invasive catheters. This research thus increases confidence in the clinical applicability and validity of this overall diagnostic monitoring approach preparatory to initial studies with human subjects.

\section{Acknowledgments}

This work was supported in part by the FNRS (Belgium), the FRST (New Zealand), the University of Liège (Crédit d'Impulsion I-03/21), the Belgian French Community (ARC - Académie Wallonie-Europe) and the University of Canterbury (UoC Targeted Scholarship Scheme). 


\section{References}

1. Angus, D.C. and M.A. Crowther, Unraveling severe sepsis: why did OPTIMIST fail and what's next? JAMA, 2003. 290(2): p. 256--258.

2. Angus, D.C., et al., Epidemiology of severe sepsis in the United States: analysis of incidence, outcome, and associated costs of care. Crit Care Med, 2001. 29(7): p. 1303-10.

3. Kumar, A., et al., Preload-independent mechanisms contribute to increased stroke volume following large volume saline infusion in normal volunteers: a prospective interventional study. Crit Care, 2004. 8(3): p. R128-36.

4. Annane, D., E. Bellissant, and J.-M. Cavaillon, Septic shock. Lancet, 2005. 365(9453): p. 63--78.

5. Bridges, E.J. and S. Dukes, Cardiovascular aspects of septic shock: pathophysiology, monitoring, and treatment. Crit Care Nurse, 2005. 25(2): p. 18-20.

6. Dellinger, R.P., Cardiovascular management of septic shock. Crit Care Med, 2003. 31(3): p. 946--955.

7. Starfinger, C., et al., Model-based identification and diagnosis of a porcine model of induced endotoxic shock with hemofiltration. Math Biosci, 2008. 216(2): p. 132-9.

8. Starfinger, C., et al., Model-based cardiac diagnosis of pulmonary embolism. Comput Methods Programs Biomed, 2007. 87(1): p. 46-60.

9. Starfinger, C., et al., Prediction of hemodynamic changes towards PEEP titrations at different volemic levels using a minimal cardiovascular model. Comput Methods Programs Biomed, 2008. 91(2): p. 128--134.

10. Starfinger, C., et al., Model-based identification of PEEP titrations during different volemic levels. Comput Methods Programs Biomed, 2008. 91(2): p. 135--144.

11. van der Kooi, T.I.I., et al., Incidence and risk factors of device-associated infections and associated mortality at the intensive care in the Dutch surveillance system. Intensive Care Med, 2007. 33(2): p. 271--278.

12. Lambermont, B., et al., Effects of endotoxic shock on right ventricular systolic function and mechanical efficiency. Cardiovasc Res, 2003. 59(2): p. 412-8.

13. Smith, B.W., Minimal haemodynamic modelling of the heart \& circulation for clinical application. 2004, University of Canterbury.

14. Smith, B.W., et al., Minimal haemodynamic system model including ventricular interaction and valve dynamics. Medical Engineering \\& Physics, 2004. 26(2): p. 131-139.

15. Chung, D.C., et al., A dynamic model of ventricular interaction and pericardial influence. Am. J. Physiol., 1997. 272(6 Pt 2): p. H2942-2962.

16. Olansen, J.B., et al., A closed-loop model of the canine cardiovascular system that includes ventricular interaction. Computers and Biomedical Research, 2000. 33: p. 260295.

17. Desaive, T., et al., Cardiovascular Modelling and Identification in Septic Shock Experimental validation. Proceedings of the 17th IFAC World Congress July 6-11, 2008, Seoul, Korea, 2008.

18. Baselli, G., et al., Model for the assessment of heart period and arterial pressure variability interactions and of respiration influences. Med Biol Eng Comput, 1994. 32(2): p. 143-52. 
19. Baselli, G., et al., Spectral and cross-spectral analysis of heart rate and arterial blood pressure variability signals. Comput Biomed Res, 1986. 19(6): p. 520-34.

20. Baselli, G., et al., Cardiovascular variability signals: towards the identification of a closedloop model of the neural control mechanisms. IEEE Trans Biomed Eng, 1988. 35(12): p. 1033-46.

21. Pagani, M., et al., Power spectral analysis of heart rate and arterial pressure variabilities as a marker of sympatho-vagal interaction in man and conscious dog. Circ Res, 1986. 59(2): p. 178-93.

22. Task Force of the American College of Critical Care Medicine, S.o.C.C.M., Practice parameters for hemodynamic support of sepsis in adult patients in sepsis. Crit Care Med, 1999. 27(3): p. 639--660.

23. Baan, J., et al., Continuous measurement of left ventricular volume in animals and humans by conductance catheter. Circulation, 1984. 70: p. 812-823.

24. Burkhoff, D., et al., Accuracy of volume measurement by conductance catheter in isolated, ejecting canine hearts. Circulation, 1985. 72(2): p. 440--447.

25. Guyton, A.C. and J.E. Hall, Textbook of medical physiology. 10th ed. 2000, Philadelphia ; London: Saunders. xxxii, 1064.

26. Starfinger, C., Patient-specific modelling of the cardiovascular system for diagnosis and therapy assistance in critical care. 2008, University of Canterbury: Christchurch, New Zealand.

\section{Figure Legends}

Figure 1: Extended CVS model overview which includes additional compartments $(P, V)_{\text {sys }}$ and $(P, V)_{\text {cap }}$ to differentiate the arterial and venous sides of the pulmonary and systemic circulation.

Figure 2: Parameter identification algorithm: 1.) a set of parameters is used for an initial simulation, 2.) data is then scaled to match the measured data and 3.) identified. This process is iterated until the simulation output is acceptable.

Figure 3 (a)-(c): Model output (dotted) vs clinical (solid line) volume and pressure waveform signals for right ventricle (RV). The upper panel shows the clinical vs. simulated ventricle and arterial pressure. The lower panel shows the clinical vs. simulated ventricle volume. The results are shown for (a) 0, (b) 90 and (c) 120 minutes into the experiment. There is no re-scaling of the results, which are the final product of resimulating the identified animal-specific model and comparing to the clinical data.

Figure 4: Clinical (solid line) vs simulated identified animal-specific model (cross) right ventricular stroke volume (RVSV) over all analyzed times and pigs.

Figure 5: Clinical (solid lines) vs simulated systolic (cross) and diastolic (box) arterial pressures (SAP, 
DAP) over all times and pigs.

Figure 6: Clinical (solid lines) vs simulated systolic (cross) and diastolic (box) pulmonary artery pressures (SPAP, DPAP) over all times and pigs.

Figure 7 (a)-(d): Mean normalized values for estimated systemic volume $V_{\text {sys,eff }}(a)$ and the identified model parameters systemic vascular resistance $R_{\text {sys }}(b)$, pulmonary vascular resistance $R_{\text {pulin }}(\mathbf{c})$ and resistance to venous return $\mathrm{R}_{\mathrm{vr}}$ (d) over all analyzed pigs during the septic shock experiment.

Figure 8: Mean normalized right ventricular end-systolic elastance $E_{\text {esrvf }}$ over all analyzed pigs during the septic shock experiment. Upper panel: clincal experimental results as obtained by [12] without the model; lower panel: results obtained with CVS model and identification process and clincally relevant limited data as presented in this study.

\section{Appendix A: CVS Model Equations}

Ventricle volumes and flows:

$$
\begin{aligned}
& V_{l v f}=V_{l v}-V_{s p t} \\
& V_{r v f}=V_{r v}+V_{s p t} \\
& V_{p c d}=V_{l v}+V_{r v} \\
& P_{p c d}=P_{0, p c d}\left(e^{\lambda p c d\left(V_{p c d}-V_{0, p c d}\right)}-1\right) \\
& P_{\text {peri }}=P_{p c d}+P_{t h} \\
& L_{a v} \dot{Q}_{a v}=P_{l v}-P_{a o}-Q_{a v} R_{a v} \\
& L_{m t} \dot{Q}_{m t}=P_{p u}-P_{l v}-Q_{m t} R_{m t} \\
& L_{p v} \dot{Q}_{p v}=P_{r v}-P_{p a}-Q_{p v} R_{p v} \\
& L_{t c} \dot{Q}_{t c}=P_{v c}-P_{r v}-Q_{t c} R_{t c}
\end{aligned}
$$

Pressures:

$$
\begin{aligned}
& P_{p u}=E_{p u}\left(V_{p u}-V_{d, p u}\right)+P_{t h} \\
& P_{p a}=E_{p a}\left(V_{p a}-V_{d, p a}\right)+P_{t h}
\end{aligned}
$$




$$
\begin{aligned}
& P_{v c}=E_{v c}\left(V_{v c}-V_{d, v c}\right)+P_{t h} \\
& P_{a o}=E_{p a o}\left(V_{a o}-V_{d, a o}\right) \\
& P_{s y s}=E_{s y s}\left(V_{s y s}-V_{d, s y s}\right) \\
& P_{c a p}=E_{c a p}\left(V_{c a p}-V_{d, c a p}\right)
\end{aligned}
$$

Volumes:

$$
\begin{aligned}
& \dot{V}_{p v}=Q_{\text {pulout }}-Q_{m t} \\
& \dot{V}_{p a}=Q_{p v}-Q_{\text {pulin }} \\
& \dot{V}_{v c}=Q_{v r}-Q_{t c} \\
& \dot{V}_{a o}=Q_{a v}-Q_{\text {sys }} \\
& \dot{V}_{\text {sys }}=Q_{\text {sys }}-Q_{v r} \\
& \dot{V}_{\text {cap }}=Q_{\text {pulin }}-Q_{\text {pulout }}
\end{aligned}
$$

Flows:

$$
\begin{array}{r}
Q_{s y s}=\frac{P_{a o}-P_{s y s}}{R_{s y s}} \\
Q_{v r}=\frac{P_{s y s}-P_{v c}}{R_{v r}} \\
Q_{\text {pulin }}=\frac{P_{p a}-P_{c a p}}{R_{p u l i n}} \\
Q_{\text {pulout }}=\frac{P_{c a p}-P_{p u}}{R_{\text {pulout }}}
\end{array}
$$

Ventricular interaction and septum volume:

$$
\begin{gathered}
P_{l v}=P_{l v f}+P_{p e r i} \\
P_{r v}=P_{r v f}+P_{p e r i} \\
P_{l v f}=d r i L \cdot E_{e s, l v f} \cdot\left(V_{l v f}-V_{d, l v f}\right)+(1-d r i L) \cdot P_{0, l v f} \cdot\left(e^{\lambda_{l v f}\left(V_{l v f}-V_{0, l v f}\right)}-1\right) \\
P_{r v f}=d r i R \cdot E_{e s, r v f} \cdot\left(V_{r v f}-V_{d, r v f}\right)+(1-d r i R) \cdot P_{0, r v f} \cdot\left(e^{\lambda_{r v f}\left(V_{r v f}-V_{0, r v f}\right)}-1\right)
\end{gathered}
$$




$$
\begin{array}{r}
e(t) E_{e s, s p t}\left(V_{s p t}-V_{d, s p t}\right)+\left(1-e(t) P_{0, s p t}\left(e^{\lambda_{s p t}\left(V_{s p t}-V_{0, s p t}\right)}-1\right)=e(t) E_{e s, l v f t}\left(V_{l v}-V_{s p t}\right)\right. \\
+(1-e(t)) P_{0, l v f}\left(e^{\lambda l v f t\left(V_{l v}-V_{s p t}\right)}-1\right)-e(t) E_{e s, r v f t}\left(V_{r v}+V_{s p t}\right) \\
-(1-e(t)) P_{0, r v f}\left(e^{\lambda_{r v f t}\left(V_{r v}+V_{s p t}\right)}-1\right)
\end{array}
$$

\begin{tabular}{|l|l|}
\hline \multicolumn{2}{|l|}{ Abbreviations used in the CVS model } \\
\hline Abbreviation & Description \\
\hline$\lambda$ & parameter in EDPVR \\
\hline$P_{0}$ & parameter in EDPVR \\
\hline$I V$ & left ventricle \\
\hline$r V$ & rRight ventricle \\
\hline$I v f$ & left ventricle free wall \\
\hline$r v f$ & right ventricle free wall \\
\hline$s p t$ & septum \\
\hline$p c d$ & pericardium \\
\hline$V_{0}$ & volume at zero pressure \\
\hline$V_{d}$ & unstressed chamber volume \\
\hline$R$ & resistance \\
\hline$E$ & elastance \\
\hline$L$ & inertance \\
\hline$P$ & pressure \\
\hline$Q$ & flow \\
\hline$V$ & volume \\
\hline$m t$ & mitral valve \\
\hline$t c$ & tricuspid valve \\
\hline$a v$ & aortic valve \\
\hline
\end{tabular}




\begin{tabular}{|l|l|}
\hline$p v$ & pulmonary valve \\
\hline pulin & systemic pulmonary \\
\hline pulout & venous pulmonary \\
\hline sys & systemic \\
\hline cap & capillary \\
\hline$v r$ & venous return \\
\hline es & end-systolic \\
\hline Pth & intrathoracic pressure \\
\hline period & heart beat period \\
\hline driL & Activation (driver) function for LV \\
\hline driR & Activation (driver) function for RV \\
\hline driS & Activation (driver) function for Septum \\
\hline
\end{tabular}

